\title{
La calidad de las IDE desde el punto de vista de la interoperabilidad
}

\author{
Antonio F. Rodríguez* \\ Francisco Javier Ariza**
}

Recibido 02 de marzo de 2018, aceptado 29 de agosto de 2018

\section{Resumen}

Las Infraestructuras de Datos Espaciales (IDE) están reconocidas, bajo la etiqueta "Servicios y Sistemas de Información Geoespacial Integrada" como uno de los objetivos definidos en UN-GGIM (2018) para alcanzar los Objetivos 2030 de Desarrollo Sostenible. Sin embargo, varias fuentes indican que las IDE no están produciendo los resultados esperados. Para facilitar que se solucionen sus problemas y deficiencias, resulta especialmente adecuado profundizar en la descripción de la calidad de las IDE existentes. En este trabajo se intenta avanzar en esa línea desde un punto de vista basado en el concepto de interoperabilidad. En primer lugar, se repasan y comentan las principales metodologías formulada hasta ahora para describir y determinar la calidad de una IDE. Se propone una clasificación, meramente descriptiva, de los indicadores utilizados para ello: indicadores de acciones realizadas, de calidad de resultados, de uso, de impacto y de coste-beneficios. Y se describe un conjunto de indicadores de calidad de los resultados, es decir de los componentes de una IDE, para caracterizarlos siguiendo el European Interoperability Framework v2 europeo establecido en 2017. Finalmente se esbozan unas conclusiones, y se proponen líneas de mejora para optimizar la situación, muchas de las cuales suponen abrir campos de investigación muy necesarios.

Palabras clave: calidad, interoperabilidad, calidad de recursos de una IDE.

* Centro Nacional de Información Geográfica (CNIG), Instituto Geográfico Nacional (IGN) de España, Calle General Ibáñez de Ibero 3, 28003 Madrid, España, correo electrónico: afrodriguez@fomento.es

** Departamento de Ingeniería Cartográfica, Geodésica y Fotogrametría, Universidad de Jaén, Campus Las Lagunillas, s/n, 23071, Jaén, España, correo electrónico: fjariza@ujaen.es 


\section{Resumo}

As Infraestruturas de Dados Espaciais (IDE) são reconhecidas como "Serviços e Sistemas de Informação Geoespacial Integrada" conforme um dos objetivos definidos no UN-GGIM (2018) para alcançar os Objetivos 2030 do Desenvolvimento Sustentável. Embora várias fontes indiquem que as IDE não estão produzindo os resultados esperados, para facilitar que se solucionem seus problemas e deficiências, resulta especialmente adequado se aprofundar a descrição da qualidade das IDE existentes. Neste trabalho se tenta avançar nessa linha desde o ponto de vista baseado no conceito de interoperabilidade. Em primeiro lugar, se repassam e comentam as principais metodologias formulada até agora para descrever e determinar a qualidade de uma IDE. Se propõe uma classificação, meramente descritiva, dos indicadores utilizados para ela: indicadores de ações realizadas, de qualidade de resultados, de uso, de impacto e de custo benefícios. E, se descreve um conjunto de indicadores de qualidade dos resultados, caracterizando os componentes de uma IDE seguindo o European Interoperability Framework v2 estabelecido em 2017. Finalmente, se esboçam umas conclusões, e se propõem linhas de melhoria para otimizar a situação, muitas das quais se supõem abrir campos de investigação muito necessários.

Palavras chave: qualidade, interoperabilidade, qualidade de recursos de uma IDE.

\section{Abstract}

The Spatial Data Infrastructures (SDIs) are recognized, under the label of "Integrated Geospatial Information Systems and Services" as one of the goals defined in UN-GGIM (2018) in order to achieve the Sustainable Development Goals 2030. Nevertheless, several voices are saying that the SDIs are not yet producing the expected results. To facilitate the resolution of their problems and gaps, it would be very useful to go deep in the existing SDI quality descriptions. This paper tries to go forward in this line of work taking as a basis the concept of interoperability as a core concept in SDIs. First of all, a brief and commented analysis is provided of the methodologies proposed until now for describing SDI quality. A descriptive taxonomy of quality indicators is proposed: actions performed indicators, quality results indicators, use indicators, impact indicators and cost-benefit indicators. A set of quality results indicators (resources implemented quality indicators) is presented following the European Interoperability Framework v2 stablished in 2017. Finally, a set of conclusions is outlined and some lines of work for improvement the situation are proposed, in most of cases with very necessary research areas associated.

Key words: quality, interoperability, SDI quality. 
Si bien sólo unos pocos son capaces de dar origen a una política, todos nosotros somos capaces de juzgarla. Pericles (alrededor del 430 a. C.)

\section{Introducción}

Las Infraestructuras de Datos Espaciales (IDE) están reconocidas, bajo la etiqueta "Servicios y Sistemas de Información Geoespacial Integrada" como uno de los objetivos definidos en UN-GGIM (2018) para alcanzar los Objetivos 2030 de Desarrollo Sostenible. Sin embargo, viven un momento de desarrollo peculiar que, en cierto sentido, puede parecer paradójico. Desde sus primeras formulaciones en la década de los noventa (Orden Ejecutiva, 1994) experimentaron una primera etapa de desarrollo que condujo a la aparición temprana de herramientas tecnológicas suficientes como para iniciar su implantación a finales de los 90. La primera versión de la norma de metadatos estadounidense apareció en 1998 (FGDC, 1998) y la versión 1.0 del estándar Web Map Service, en el 2000 (OGC, 2000).

Sin embargo, a pesar de las dos décadas transcurridas y de que la mayoría de los países han abierto un geoportal nacional de su IDE, muchos expertos expresan un cierto desaliento, que se podría llamar desaliento geográfico, porque no siempre se consigue rentabilizar la tecnología y las aplicaciones finales, basadas en servicios web, no "florecen" todo lo que sería deseable.

Efectivamente, el informe titulado Summary Report on the Status of implementation of the INSPIRE Directive in EU (Comisión Europea, 2017b) concluye que “...se necesitan esfuerzos adicionales a nivel de la Unión Europea y de los estados miembros para salvar los huecos existentes en la implementación y recoger todos los beneficios de la Directiva". Por otro lado, el lema de la Conferencia INSPIRE 2017, Thinking out of the box ("Una invitación a pensar desde una nueva perspectiva"), y el título de una de las sesiones, What if...? (“¿Y si...?”), parecen síntomas de una búsqueda urgente de soluciones y en un plano más técnico y concreto, el documento "Spatial Data On the Web Best Practices" (W3C, 2017), elaborado por un grupo de trabajo conjunto de expertos del World Wide Web Consortium (W3C) y del Open Geospatial Consortium (OGC), que trata de aconsejar sobre cómo aplicar mejor las tecnologías web en las IDE, constituye un intento explícito de encontrar esas soluciones.

Es una situación que recuerda lo que ocurría con los Sistemas de Información Geográfica (SIG) a principios de la década de los noventa: una tecnología que prometía mucho, pero que no acababa de alcanzar su madurez (Rodríguez, 1993), que llegaría una década después, en los años 2000, cuando ISO/TC 211 aportó las soluciones esperadas en forma de modelos, formatos, metadatos, calidad y especificaciones establecidos en la familia de normas ISO 19100 (Ariza y Rodríguez, 2008). 
Esa posible inmadurez en el caso de las IDE, cuya complejidad resulta obvia si se considera su concepción como sistema de sistemas (Béjar, 2009), se debe en nuestra opinión no tanto a sus componentes más técnicos, ya que hay estándares, software libre disponible y herramientas más que suficientes para su implementación, como a sus aspectos organizativos. Si se tiene en cuenta que las IDE operan en un entorno globalizado y la amplitud y heterogeneidad de actores que deben coordinarse para su correcto funcionamiento, es lógico que factores como el apoyo político sostenido y al más alto nivel posible, la financiación, la sostenibilidad, rentabilidad, y sobre todo, su gobernanza y coordinación, resulten de gran importancia (Maganto et al., 2012).

Muy probablemente haya que esperar una década más, como ocurrió con los SIG, para ver cómo las IDE se extienden y dan todos sus frutos. Sin embargo, mientras que los SIG que han ido apareciendo, han demostrado una notable flexibilidad al ir incorporando las nuevas tecnologías (Orientación a Objeto, computación en la nube, 3D...), las IDE no están incorporando con la misma facilidad las tecnologías de vanguardia (Datos enlazados, BIM, Smart Cities, Big Data, Realidad Virtual/Aumentada...) y la brecha entre la interoperabilidad en el laboratorio y en el sector productivo parece no cerrarse nunca.

En cualquier caso, el establecer un modelo de calidad IDE puede contribuir a monitorizar su progreso, identificar áreas de mejora, potenciar la sana competitividad entre proyectos y servir así de palanca para rentabilizar la tecnología.

Para considerar la calidad de una IDE, en este trabajo se va a ofrecer una panorámica de los métodos abordados hasta ahora, para tomar luego como punto de partida el modelo de interoperabilidad europeo. La interoperabilidad es el concepto clave en que se basan las IDE, tal y como se define en el European Interoperability Framework v2 (EIFv2) (Comisión Europea, 2017). Es uno de los pilares básicos en uno de los ámbitos legislativos más complejos y desarrollados, la Unión Europea, que ha dado lugar a una IDE supranacional bajo la Directiva INSPIRE (2007/2/CE).

De esta forma, el objetivo de este trabajo es revisar de una manera crítica los conceptos actuales relativos a la calidad de una IDE, como aspecto clave para estimular y potenciar su desarrollo. Como consecuencia final, se propone profundizar en la caracterización de la calidad de todos los componentes de una IDE, para lo que se identifican líneas de investigación necesarias para poder progresar en esa línea de trabajo.

\section{Calidad de una IDE}

Existe una amplia variedad de estudios y documentación (Vancauwenberghe et al., 2018, Kelm, 2017, Welle Donker y van Loenen, 2016 y Randolf Pérez et al., 2015), en los que se aborda la caracterización de la calidad una IDE mediante la definición de un conjunto de indicadores. Hay que advertir que para establecer adecuadamente 
un sistema de indicadores, de acuerdo a la norma UNE 66175:2003 (Guía para la implantación de sistemas indicadores) y a la ISO 19157: 2013 sobre calidad de datos geográficos, es necesario definir cuatro elementos para cada indicador: qué aspecto vamos a evaluar (por ejemplo, la exactitud posicional absoluta), qué medida vamos a emplear (por ejemplo, el Error Medio Cuadrático en distancia), qué metodología se va a aplicar (se toman cuatro puntos por $\mathrm{km}^{2} \ldots$... y cómo se va a expresar el resultado final, por ejemplo, como un resultado numérico $(3 \mathrm{~m})$ o como un "pasa/no pasa" un umbral de calidad prefijado (si es menor o no que $3 \mathrm{~m}$ ).

Fundamentalmente, todas las metodologías se basan en la definición de un conjunto de indicadores, como puede verse en la amplia variedad de estudios y trabajos recopilados en la obra Multi-View Framework to Asses SDIs (Crompvoets et al., 2008) y en Morera et al. (2012), que se pueden agrupar a efectos meramente descriptivos en cinco clases de indicadores, que se expondrán a continuación con más detalle: indicadores de acciones realizadas, de calidad de resultados, de uso, de impacto y de coste-beneficio.

1. Indicadores de acciones realizadas

Son indicadores numéricos y objetivos que describen las acciones realizadas, como por ejemplo, el número de recursos implementados y sus características. Son útiles para fijar una línea base y poder monitorizar la evolución del esfuerzo de inversión realizado a lo largo del tiempo. Así son la mayoría de los indicadores definidos en el marco INSPIRE por medio de los llamados indicadores de seguimiento anual (Tabla 1) definidos en (Comisión Europea, 2009).

Tabla 1

Principales indicadores de acciones realizadas INSPIRE

\begin{tabular}{|c|c|}
\hline Indicador & Definición \\
\hline \multicolumn{2}{|c|}{ Indicadores de conjuntos de datos espaciales (CDE) } \\
\hline $\mathrm{DSv}$ & $\mathrm{N}^{\mathrm{o}}$ de CDE reportados \\
\hline DSil & $\%$ medio de superficie nacional cubierta por los CDE \\
\hline \multicolumn{2}{|r|}{ Indicadores de servicios } \\
\hline SDs & $\mathrm{N}^{\mathrm{o}}$ total de servicios de datos espaciales \\
\hline $\begin{array}{l}\mathrm{NSv} 1,2 \\
3,4,5\end{array}$ & $\begin{array}{l}\mathrm{N}^{\mathrm{o}} \text { de servicios de búsqueda, visualización, descarga, } \\
\text { transformación e invocación }{ }^{1}\end{array}$ \\
\hline
\end{tabular}

1 Los servicios de invocación sirven para hacer llamadas a servicios no INSPIRE y transformar la respuesta al vuelo de manera que el resultado sean servicios conformes a INSPIRE. 
Continuación Tabla 1

\begin{tabular}{cl}
\hline Indicador & \multicolumn{1}{c}{ Definición } \\
\hline NSi1.1 & \% de CDE con metadatos cargados en un catálogo \\
NSi1.2 & $\begin{array}{l}\text { \% de servicios con metadatos cargados en un catálogo } \\
\text { NSi2 de CDE accesibles vía servicios de visualización y } \\
\text { descarga }\end{array}$ \\
\hline & Indicadores de existencia metadatos \\
\hline MDi1.4 & $\%$ de CDE con metadatos \\
MDi1 & $\%$ de CDE y servicios con metadatos \\
\hline
\end{tabular}

Son indicadores fáciles de determinar, simplemente inventariando los recursos implementados. Otros sistemas de indicadores de este tipo son, por ejemplo, los definidos por Eelderink y Steidler (Crompvoets et al., 2008).

Tienen el gran inconveniente de que sirven para cuantificar el esfuerzo de inversión realizado, pero no si el esfuerzo va en la dirección adecuada, si los recursos implementados tienen la calidad necesaria ni si el impacto final es el deseado. Por lo tanto su utilidad depende de que se complementen con indicadores de los otros tipos.

Por otro lado, la interpretación de sus valores debe hacerse muy cuidadosamente, especialmente los que no se expresan mediante un tanto por ciento, ya que dependen de varias circunstancias. No resulta fácil comparar los valores de algunos de ellos entre unos países y otros, como por ejemplo el número de servicios, porque en algunos estados miembros los servicios pueden estar muy atomizados, por escalas e incluso por tipo de objeto geográfico, mientras que en otro puede ser que solo se reporte un servicio para la mejor resolución y para todos los datos. Algo parecido ocurre con el número de conjuntos de datos; hay países que reportan cualquier CDE de los temas INSPIRE, aunque sea de un solo municipio, mientras que otros solo reportan los CDE fundamentales para todo el país que mejor describen la realidad nacional. Para complicar más aún la situación, algunos estados han cambiado de criterio a lo largo del tiempo, con lo que resulta evidente la necesidad de unificar criterios y mantenerlos en el tiempo para que los valores y su evolución sean interpretables. Véanse Vandenbroucke (2012) y European Environmental Agency (2014).

2. Indicadores de calidad de resultados

Se basan en la descripción de la calidad de los resultados de las acciones realizadas, en el caso de la IDE, esto se traduce en calidad de los recursos implementados: datos, metadatos y servicios. Los indicadores INSPIRE de seguimiento 
anual que describen la conformidad con los Reglamentos europeos de conjuntos de datos, metadatos y servicios son indicadores de este tipo (Tabla 2).

Tabla 2

Principales indicadores de calidad de resultados INSPIRE

\begin{tabular}{ll}
\hline Indicador & \multicolumn{1}{c}{ Definición } \\
\hline DSi2 & \% de conjuntos de datos espaciales (CDE) \\
conformes \\
NSi4 & \% de servicios conformes \\
MDi2.4 & \% de servicios con metadatos conformes \\
MDi2 & \% de CDE y servicios con metadatos conformes \\
\hline
\end{tabular}

En este tipo de indicadores es en el que se va a centrar este artículo, considerando la calidad de un amplio abanico de componentes de una IDE, más allá de datos, metadatos y servicios.

3. Indicadores de uso

Evalúan la utilización de los recursos de las IDE, no solo mediante indicadores cuantitativos de los volúmenes de consultas y tráficos de datos, sino también evaluando el número de usuarios, organizaciones, aplicaciones y recursos de valor añadido que se generan. En esa línea están los indicadores definidos en la Directiva INSPIRE que se recogen en la Tabla 3 y los incluidos en otros estudios similares, como Crompvoets (2008).

Tabla 3

Principales indicadores INSPIRE de uso

\begin{tabular}{ll}
\hline Indicador & \multicolumn{1}{c}{ Definición } \\
\hline NSi3 & $\mathrm{N}^{\text {o }}$ medio de peticiones anuales por servicio \\
$\mathrm{NSi3} .1$ & $\begin{array}{l}\mathrm{N}^{\text {o }} \text { medio de peticiones anuales por servicio de } \\
\text { búsqueda } \\
\mathrm{N}\end{array}$ \\
$\mathrm{N}$ o medio de peticiones anuales por servicio de \\
visualización \\
$\mathrm{NSi3.3}$
\end{tabular}


De nuevo, es necesario interpretar adecuadamente los valores de este tipo de indicadores y su evolución. Por ejemplo, la implementación de unos servicios de descarga excelentes puede disminuir el $\mathrm{n}^{\mathrm{o}}$ de peticiones de visualización y viceversa. Por otro lado, los Web Map Tiles Service (WMTS) exigen un alto número de peticiones frente a los Web Map Service (WMS) para un mismo caso de uso. De cualquier manera, este tipo de indicadores es aconsejable para analizar la evolución en el tiempo del uso de los servicios implementados.

4. Indicadores de impacto

Tratan de evaluar la eficacia o grado en el que una IDE alcanza sus últimos objetivos, en función del resultado o impacto esperado por los usuarios o por la sociedad (Giff, 2008). En esta categoría entrarían todos los indicadores que describen de una manera u otra la satisfacción del usuario. En este caso, la dificultad estriba en caracterizar ese impacto esperado, lo que puedo hacerse a bajo nivel de abstracción eligiendo un resultado concreto, por ejemplo el aumento de la cifra de negocio global del sector de la Información Geográfica (IG) o la disminución de costes de los proyectos que explotan IG o a alto nivel de abstracción, como la aportación de las IDE a la consecución de los objetivos de la Agenda 2030 de Naciones Unidas.

Su definición depende de los objetivos y planteamientos estratégicos que se tengan y parecen, en cierta medida, más relevantes porque describen las consecuencias y efectos ocasionados por la implementación de una IDE. Al mismo tiempo, aunque se definan como algo tan simple como la satisfacción de usuario, pueden en parte reflejar la influencia de otros factores que confluyen, como las expectativas previas, de manera que resulta difícil aislar el impacto de la implementación de una IDE de otras circunstancias económicas, sociales y de todo tipo que pueden influir en sus efectos.

5. Indicadores de coste-beneficio

Son indicadores que intentan reflejar la eficiencia de los recursos implementados, es decir el resultado de un balance coste-beneficio, que a menudo es lo más convincente y significativo para tomadores de decisiones y responsables de efectuar inversiones públicas. Es ésta una línea de trabajo difícil y siempre con resultados incompletos debido a que gran parte de los beneficios de las IDE son intangibles y difusos, que muchas veces se producen a muy largo plazo y que resulta imposible comparar en la práctica cómo evoluciona económicamente un mismo país sin y con IDE. Sin embargo, hay un buen número de estudios parciales que arrojan tasas de coste-beneficio considerablemente altas, como Morera et al. (2012), Balciunas et al. (2016) y de Jong (2016). 
Hay que hacer notar que, si bien los indicadores de acciones realizadas y uso tienen la ventaja de ser más fácilmente definibles como parámetros cuantitativos acompañados de métodos objetivos de determinación, son menos interesantes, en cuanto a su significado, que los indicadores de impacto y los de coste-beneficio. Efectivamente, se puede tener un amplio abanico de servicios web implementados, muy usados, por ejemplo como cartografía fundamental de fondo, pero que aportan pocos servicios de valor añadido y escasos casos de uso con un impacto real y un beneficio notable. Por el contrario, los indicadores de impacto suelen dar lugar a parámetros cualitativos de determinación difícilmente objetivable, mientras que los de coste-beneficio aunque son obviamente cuantificables, son de muy difícil evaluación, dado que la mayoría de los beneficios de las IDE son intangibles y se dan a largo plazo. Sin embargo, estos dos últimos tipos parecen más interesantes porque describen los efectos y consecuencias prácticas de la implementación de una IDE.

Los indicadores de segundo tipo, los de calidad de resultados de las acciones ejecutadas, en suma, los de calidad de los componentes de una IDE, precisan de cierta dedicación de recursos, por contemplar aspectos técnicos a veces sofisticados, sin embargo tienen la ventaja de que pueden relacionarse, como luego se verá, con la interoperabilidad, fundamento y concepto clave en las IDE.

Su determinación es más fácilmente objetivable que los indicadores de impacto $\mathrm{y}$ de coste-beneficio. Por otro lado, aunque a primera vista puedan parecer poco significativos, como los indicadores de acciones realizadas o los de uso, si se define un abanico suficiente amplio y comprensivo de componentes de una IDE, incluyendo tanto aspectos técnicos como organizativos, su calidad puede ser clave y tanto el impacto como el balance coste-beneficio resultarán ser consecuencias de esa calidad.

Tienen además la ventaja de que, al contrario que los indicadores de los otros cuatro tipos, no dependen tanto de la variedad de planteamientos y modelos que se pueden llevar a cabo para la implementación de la IDE, lo que evita la variedad de experiencias que dificulta la convergencia y consenso en un planteamiento común que permita comparar la calidad de varias IDE diferentes. Hay que pensar que para afinar y validar objetivamente un sistema de indicadores que no sea del segundo tipo, habría que mantenerlo en el tiempo y repetir su ejecución para poder extraer conclusiones, lo que supondría inversiones y esfuerzos considerables.

Por lo tanto, los indicadores del segundo tipo son los que permiten comparar más fácilmente IDE diferentes.

\section{Una alternativa: la interoperabilidad como indicador de calidad}

En lo que sigue se esboza un planteamiento que parte de un punto de vista alternativo basado en la interoperabilidad, una de las ideas clave de las IDE, que identifica la calidad de una IDE con ese concepto y por lo tanto, con la calidad de sus compo- 
nentes, expresada mediante indicadores del tipo 2, lo que permite una caracterización más objetiva y comparable, basada en aspectos técnicos que complementan a otros más organizativos.

Para ello, se tomará como base el European Interoperabiliy Framework v2 (Comisión Europea, 2017) (EIFv2), tal y como se plantea en Rodríguez et al. (2017). Ese modelo de interoperabilidad considera cuatro niveles:

- Interoperabilidad legal, que se encarga de asegurar qué organizaciones de las que trabajan en diferentes marcos, políticas y estrategias legales pueden interoperar sin encontrar barreras legislativas, independientemente de que haya o no un Marco Legal de ámbito superior que las acoja.

- Interoperabilidad organizacional, que se ocupa de la coordinación de procesos, responsabilidades y expectativas de las organizaciones para que puedan interoperar sin disfuncionalidades.

- Interoperabilidad semántica, que en realidad incluye (a) la interoperabilidad semántica propiamente dicha, que se ocupa del significado de lo que se transmite en cada momento, y (b) la interoperabilidad sintáctica, que se encarga de la interoperabilidad entre formatos físicos de datos y metadatos.

- Interoperabilidad técnica, que cubre la interconexión de la infraestructura de comunicaciones subyacente y de aplicaciones, es decir servicios web. Es la interoperabilidad más ligada a las Tecnologías de la Información y las Comunicaciones y a menudo es el único nivel de interoperabilidad que se considera en algunos estudios como en Nativi et al. (2012).

Cada uno de estos niveles conlleva sus propios aspectos de la calidad, conceptualmente diferentes a la hora de diseñar un modelo de calidad de una IDE. Teniendo en cuenta una de las definiciones clásicas de IDE (GSDI, 2012): "Tecnologías, políticas, estándares y recursos humanos necesarios para adquirir, procesar, almacenar, distribuir y mejorar la utilización de datos geoespaciales" (traducción propia), se van a generalizar los niveles de interoperabilidad del EIFv2 para tener en cuenta todos los componentes de una IDE en un sentido amplio e incluir así todos los posibles factores.

\section{Interoperabilidad legal}

Considerando que depende fundamentalmente de la calidad del Marco legal definido en el ámbito en el que interoperan los componentes de una IDE, aparecen situaciones problemáticas derivadas a menudo de la interacción de dos dominios tan heterogéneos en principios y métodos como son el legal y el tecnológico. Por ejemplo, los procesos de definición y reforma de disposiciones legales son largos, pesados y, a menudo, de resultado incierto comparados con los procesos de generación 
de especificaciones técnicas. Otra diferencia radica en que el ámbito legal está más fragmentado por países que el tecnológico. A continuación, se enumeran las principales situaciones problemáticas que se pueden presentar en este nivel:

- Marco legal incompleto. Aparece cuando el marco legislativo de una IDE no contempla y regula claramente todos los aspectos que sólo pueden ser definidos legalmente y resultan imprescindibles para el buen funcionamiento de una IDE. Esa falta de completitud puede deberse a que los textos legales

- sean demasiado laxos,

- estén desenfocados y se concentren en otros temas relacionados con las

IDE, pero que no resulten centrales,

- simplemente, no existan,

con lo que toda la función regulatoria recae en documentos técnicos de cumplimiento voluntario.

- Marco legal demasiado rígido. En el difícil equilibrio entre el conocimiento legal y el técnico, este riesgo aparece a menudo cuando el legislador invade campos que evolucionan con una rapidez muy grande en comparación con los tiempos necesarios para modificar una disposición legal, lo cual depende parcialmente de la mayor o menor agilidad que tenga el poder legislativo en cada país o ámbito regulatorio. Sin embargo, parece razonable que, dada la brecha temporal existente entre la evolución de la tecnología y la de las disposiciones legales, el Marco legal contemple tan sólo aspectos conceptuales, de principios muy generales, y puramente competenciales y organizativos, sin entrar nunca en aspectos relacionados con las TIC y las interoperabilidades semántica y técnica, que deben estar regulados por documentos técnicos mucho más dinámicos.

Un ejemplo de la situación descrita es la que se da en al marco de la implementación de la Directiva europea INSPIRE. Por un lado, se ha aprobado un conjunto de Reglamentos europeos, de obligado cumplimiento en todo el territorio de la UE, que descienden a especificar aspectos técnicos acerca de datos, metadatos y servicios. Por otro lado, existen una serie de Directrices técnicas que ayudan a cumplir los anteriores.

Los Reglamentos son excesivamente rígidos, no admiten una adaptación suficientemente ágil a una tecnología en continua evolución. Hay que mencionar que desde la aparición de la Web 2.0 en el año 2004, se habla de la versión beta perpetua, como filosofía de desarrollo flexible y continuamente adaptable $^{2}$ en oposición a la liberación de versiones del software cerradas y completas.

Por otro lado, las Directrices, aunque son fácilmente adaptables, no son obligatorias, con lo que tampoco está asegurada completamente la interoperabilidad. 
Quizás la solución ideal sería un Marco legal que estableciera la obligatoriedad de cumplir las últimas versiones en cada momento de un conjunto de especificaciones técnicas publicadas en la web y mantenidas por un organismo competente por ley para mantenerlas al día y que funcionase de manera abierta para todas las partes interesadas y tomase decisiones por consenso o por votación, a imagen y semejanza de las organizaciones de estandarización, como el OGC o el W3C.

- Contenido legal de baja calidad. Formalmente los textos legales pueden ser farragosos, ininteligibles, contradictorios, faltos de lógica, innecesariamente complejos y complicados, incluso lingüísticamente pobres y deficientes. Aspectos todos ellos que pueden dar lugar a interpretaciones erróneas o divergentes y pérdidas de tiempo invertido en encontrar la interpretación correcta de la ley. Aparece aquí la dificultad para definir parámetros de calidad y métodos de determinación que describan cuantitativamente la calidad del contenido del Marco legal.

- Mala accesibilidad del Marco legal. En ocasiones el Marco legal de una IDE puede ser fragmentario, carecer de textos consolidados o resultar poco accesible, circunstancia esta última que afecta aproximadamente el $10 \%$ de los países ${ }^{3}$.

La fragmentación del Marco legal de una IDE podría cuantificarse mediante el número de disposiciones legales que es necesario consultar para implementar un nodo IDE completo. En el caso de la IDE de la Unión Europea (INSPIRE), el Marco legal específico está formado en el 2018 por una Directiva Europea y trece Reglamentos europeos, lo que hace un total de catorce disposiciones legales.

- Falta de garantías de aplicabilidad del Marco legal. En ocasiones se dispone de un Marco legal adecuado y completo, pero no se dispone de herramientas que permitan hacer efectivo su cumplimiento y se depende de la buena voluntad de los integrantes de la comunidad IDE para asegurarlo. Ese factor depende mucho de aspectos culturales, pero siempre es necesario disponer de algún tipo de modelo de evaluación y régimen sancionador para las organizaciones que no cumplan el Marco legal, que actúe como disuasión e incentive su cumplimiento riguroso y diligente.

Después de todo lo dicho se podría afirmar que de manera resumida las siguientes directrices podrían definir un Marco legal de calidad que facilite la interoperabilidad:

- Un Marco legal puramente teórico, organizativo y competencial, que se limite a establecer los principios y conceptos básicos (interoperabilidad, estándares abiertos, datos y servicios abiertos, servicio, metadatos, geoportal, etc.), asig- 
nando una serie de competencias (coordinación de la IDE, órgano ejecutivo, procedimientos de participación y toma de decisiones) y una arquitectura territorial y temática (basada en nodos de referencia).

- Un Marco legal que no especifique aspectos técnicos, sino que establezca un conjunto de especificaciones técnicas de obligado cumplimiento, definidas por una organización creada $a d$ hoc, de carácter abierto, democrático y participativo.

- Un conjunto de procedimientos técnicos claros y transparentes relacionados con su definición, mantenimiento y actualización.

- Un conjunto de mecanismos claros y razonables para asegurar su cumplimiento (modelo de evaluación y régimen sancionador).

No se conocen mecanismos para verificar si el Marco legal de una IDE es suficientemente flexible o no para su desarrollo. Una vía que parece factible, consistiría en analizar si es posible de una manera fácil, no traumática y en un tiempo aceptable su completa adaptación a una tecnología completamente nueva y diferente, como la de los datos enlazados (linked data).

La lista mencionada de los aspectos individuales de la calidad de un Marco legal mencionados, no se ha incluido como una relación exhaustiva y completa, sino como una primera aproximación sobre la que es necesario investigar y profundizar. Entre ellos los hay que parecen de difícil cuantificación, de modo que a lo sumo, permiten estimaciones cualitativas teñidas siempre de cierta subjetividad, por lo que parece que sería necesaria también la investigación en este campo. En cualquier caso, si no se progresa en esta dirección suficientemente, es muy probable que las IDE existentes se resientan, ya que el Marco legal es actualmente uno de sus puntos débiles, tal y como evidencian la variedad y heterogeneidad existente de este componente de un país a otro y la falta de criterios establecidos sobre su calidad. La existencia del Marco legal INSPIRE y de los logros y beneficios que ha supuesto, en una comunidad tan compleja lingüística y culturalmente como la europea, constituye un ejemplo inspirador para alcanzar el establecimiento de un Marco legal o recomendación para Latinoamérica, donde instituciones supranacionales como el IPGH ya han alcanzado resultados muy positivos en proyectos similares organizativamente, como el Proyecto SIRGAS, ${ }^{4}$ el Mapa Integrado de América Central (Norori Solís et al., 2013) y el Mapa Integrado Andino del Norte (Martín et al., 2017).

$4 \quad \mathrm{http} / / /$ www.sirgas.org/es/ 


\section{Interoperabilidad organizacional}

Como ya se ha mencionado, la interoperabilidad organizacional se ocupa de la coordinación de procesos, responsabilidad y expectativas de las organizaciones para que puedan interoperar sin disfuncionalidades. El riesgo de falta de interoperabilidad organizacional en una IDE incluye todos los aspectos no estrictamente técnicos relacionado con la organización, coordinación y liderazgo de una IDE. Son aspectos comunes con otros proyectos en los que interviene una red de actores independientes que deben colaborar activamente para el éxito del proyecto. Los aspectos a considerar se pueden agrupar en tres apartados:

1. Aspectos organizativos estructurales

2. Aspectos operativos organizacionales

3. Aspectos técnico-organizativos

1. Aspectos organizativos estructurales

- Apoyo político. El primer aspecto importante a considerar es el apoyo político al más alto nivel posible. La existencia de ese apoyo claro, sostenido y efectivo a la implantación de tecnologías IDE resulta clave, puesto que de él dependen la dedicación de todo tipo de recursos, humanos, inversión en formación, equipamiento, etcétera, y la existencia de una presión externa que demanda y espera resultados relevantes y útiles en un plazo de tiempo razonable.

- Existencia de un staff. El siguiente aspecto a considerar es la existencia de un staff sostenible y estable en el tiempo, que no tiene porqué ser muy numeroso, de expertos dedicados a la coordinación y liderazgo de la IDE a tiempo completo o al menos como responsabilidad principal. Eso hace posible la planificación, el diseño de la arquitectura global y la toma de decisiones de alto nivel que luego se contrastan con los resultados y permiten aprender de la experiencia. Ese "núcleo duro" de liderazgo, debe estar rodeado de un comité en el que estén representados los responsables técnicos a alto nivel de los nodos que componen la IDE a fin de que puedan consensuar todas las decisiones a llevar a la práctica.

- Comunidad de actores. Como consecuencia del aspecto anterior, debe existir una comunidad de actores que sostiene la IDE equilibrada y participativa, formada por organizaciones públicas, empresas privadas, universidades y usuarios de la infraestructura, con un espíritu de colaboración amplio y asegurado. La colaboración debe ser ágil y exenta de burocracias entre los distintos actores. 
- Dentro de esa comunidad de actores debe existir un conjunto de Grupos de Trabajo, denominados con ése o con otro nombre, que aborden y resuelvan cuantas tareas técnicas se identifiquen como necesarias para el buen funcionamiento de la IDE en su conjunto.

- Calidad de usuarios: Por último, habría que incluir aquí la calidad de los usuarios de la IDE, como actores con un papel activo de una IDE, teniendo en cuenta su implicación, la retroalimentación que devuelven, su participación activa en la toma de decisiones, incluso en procesos de crowdsourcing.

2. Aspectos operativos organizacionales

No solo es necesario considerar la calidad de una serie de estructuras e instancias organizativas, como se ha abordado en el punto anterior, teniendo en cuenta su composición, funcionamiento régimen de reuniones, representatividad y otros factores similares, sino también la calidad de los resultados que producen, lo que asegura la robustez del modelo de calidad al incorporar todas las perspectivas posibles, por ejemplo:

- La planificación de la progresiva implementación de la IDE, su evolución técnica y su aplicación a todos los sectores de actividad.

- El Plan de Medios o plan de sostenibilidad económica que asegure la financiación necesaria para que la IDE sea sostenible en el tiempo.

- La política de estándares, que engloba aspecto como:

- los estándares elegidos,

— la política de versiones y

- las medidas para verificar y conseguir que la política de estándares establecida se cumpla.

- Las recomendaciones de armonización, que van un paso más allá de los estándares de interoperabilidad seleccionados, eligiendo algunas opciones que facilitan la interoperabilidad real y práctica, como los Sistemas de Referencia de Coordenadas, un perfil de metadatos, sistemas de teselado, escalas, palabras clave, etcétera.

- El Plan de Formación y, en general, la política de recursos humanos, considerada a nivel global de la IDE.

- Las medidas para compartir software y desarrollos cuando sea posible. En un mundo en el que el modelo de producción basado en el software libre presenta ventajas claras en cuanto a reutilización del código, las aplicaciones, compartición de las inversiones y desarrollo, escalabilidad sin coste de licencias, parece lógico planificar y coordinar el desarrollo colaborativo de soluciones. 
- Los mecanismos de difusión de la información, tanto de arriba hacia abajo (información, noticias, blogs, redes sociales, feeds...) como de abajo hacia arriba (retroalimentación de usuarios y técnicos).

- Un aspecto clave en el funcionamiento de la comunidad es la eficacia con la que los proveedores de servicios capturan e incorporan la retroalimentación de sus usuarios para identificar sus necesidades y requerimientos.

3. Aspectos técnico-organizativos

Uno de los aspectos de la calidad de una IDE que entra dentro de lo organizativo, pero que es algo a la vez técnico y práctico, es la conexión y visibilidad de los nodos IDE, con una coordinación que evite la existencia de "zonas de sombra" temáticas o geográficas en las que existan recursos IDE (geoportales, datos, metadatos y servicios) que no tienen visibilidad en los Nodos IDE de Referencia de nivel superior.

Otro aspecto importante del mismo tipo es la interoperabilidad de los componentes esenciales que permiten que la IDE se comporte globalmente como un sistema único como: la navegabilidad siguiendo la red de nodos de la IDE, la coordinación de catálogos (harvesting), la arquitectura lógica de componentes, la política de Identificadores Persistentes (PID), o las medidas de verificación de la conformidad (validadores).

Por último, hay recursos básicos (servicios de catálogo, registros, codelists, sistemas de identificadores únicos, etc.), que resultan clave para que la arquitectura de la IDE pueda funcionar correctamente en la práctica.

Mención especial merece la calidad de las políticas de datos abiertos existentes. El informe (EEA-JRC, 2014) concluyó que existe una clara sinergia y correlación entre los datos abiertos y el grado de desarrollo de las IDE en un país o región. Los países europeos en los que hay datos más abiertos son los que más lejos están llegando en la implementación de INSPIRE y viceversa. Para evaluar la calidad de este aspecto pueden establecerse varios escalones de calidad creciente relacionados con la política de datos:

- Tener adoptada una política de datos de manera clara, consciente y responsable. Eso evitaría las vueltas atrás en la liberación de datos, que suelen ser mal aceptadas por los usuarios y crean inseguridad ante la sostenibilidad de las condiciones de uso.

- Mantenerla en el tiempo y adoptar medidas que garanticen su sostenibilidad, como consecuencia del punto anterior.

- Publicitarla adecuadamente, idealmente debería ser imposible que un usuario descargase un conjunto de datos sin que fuera informado de la licencia y condiciones de uso correspondientes. 
- Utilizar licencias implícitas, cuyo mero uso implica su aceptación, como ocurre habitualmente en la mayoría de bienes que adquirimos. Eso facilita los trámites para el uso de los datos y elimina una barrera tan artificial como que el usuario tenga que estar de acuerdo en las condiciones de uso.

- Utilizar licencias tipo o estándar, que garanticen la interoperabilidad de licencias. El problema que genera el hecho de que los productores de datos utilicen licencias propias, descritas en un texto ad hoc, es que, si un usuario mezcla dos conjuntos de datos para generar una obra derivada y cada uno tiene su propia licencia no estándar, es en general extraordinariamente difícil averiguar cómo se mezclan las condiciones de uso de ambas licencias.

- Definir los datos como datos abiertos, en el sentido que define la Open Knowledge Internacional (2015), la definición más completa y nítida que conocemos. Eso incluye conceptos clave como no discriminar a ningún grupo de usuarios y una eliminación radical de todo tipo de barreras técnicas, legales y económicas para el uso de los datos.

Recientemente ha aparecido un estudio interesante que extiende el concepto de apertura y propone un conjunto de catorce indicadores, con tres valores de calidad (baja, media y alta) para caracterizar la apertura de una IDE en su conjunto:

1. Estrategia y visión de datos abiertos

2. Política de datos abiertos

3. Proceso de toma de decisiones sobre política de datos abierto a actores no gubernamentales

4. Apertura a incorporar datos no gubernamentales

5. Encontrabilidad de datos en buscadores estándar

6. Difusión de datos en los portales nacionales de la IDE y de datos abiertos

7. Multilingüismo de los metadatos

8. Disponibilidad de datos sin registro de usuarios

9. Gratuidad de los datos

10. Publicación de los datos mediante servicios de visualización y descarga

11. Licencias de datos abiertas e interoperables

12. Nivel de interoperabilidad de datos

13. Casos de uso no gubernamentales

14. Estudios de beneficios de los datos abiertos

El planteamiento está demasiado centrado en los datos, cuando en realidad las IDE están orientadas a servicios; mezcla en la descripción de la apertura de una IDE indicadores de coste-beneficios, que parecen demasiado heterogéneos y pertenecientes a una categoría diferente, y no considera algunos aspectos (apertura de ser- 
vicios, apertura a la Información Geográfica Voluntaria, servicios de procesamiento, nomenclátor, sensores, etc.) que parecen también importantes. Por último, la evaluación de indicadores tan generales adolece de cierta subjetividad, problema que suele presentarse cuando se establecen indicadores cualitativos y no directamente medibles (Vancauberghe et al., 2018).

La calidad de los aspectos relacionados con la interoperabilidad organizacional puede ser descrita con uno o más elementos de calidad como los mencionados, todos ellos difíciles de cuantificar, para los que habría que definir un sistema de indicadores especificando con precisión, como ya hemos dicho, el aspecto a evaluar, el indicador elegido y el método de medida, tal y como establecen las normas en ese campo, como la mencionada UNE 66175:2003 (AENOR, 2003).

Es necesario realizar más investigación basada en la propuesta de modelos de indicadores, basados en estos aspectos y su aplicación, para poder realizar estudios comparativos y poder progresar en la selección de los esquemas más eficaces y descriptivos.

\section{Interoperabilidad semántica}

Como ya se ha indicado, la interoperabilidad semántica incluye el aspecto sintáctico y el puramente semántico. En este subapartado se tratarán ambos.

Si bien los aspectos de interoperabilidad legal y organizacional se pueden aplicar con propiedad en el ámbito completo de una IDE, los que se van a considerar bajo el aspecto de interoperabilidad semántica pueden tenerse en cuenta para una IDE, tanto en su totalidad, como para cada uno de los nodos que la conforman, e incluso para cada recurso concreto en particular. La esencia de la interoperabilidad semántica de datos radica en que el destinatario de la información en un intercambio de datos, los pueda interpretar de manera correcta, de modo que obtenga la misma información que el productor de datos quiso representar en ellos (Comisión Europea, 2017). Como ya se ha indicado, eso depende esencialmente de dos factores: que los datos se interpreten correctamente para obtener la información deseada, con el significado pretendido, lo cual se llama interoperabilidad de significado, y que la sintaxis de formatos sea correcta, que llamaremos como es habitual, interoperabilidad sintáctica.

\section{Interoperabilidad de sintáctica}

La interoperabilidad sintáctica está asociada a la ausencia de problemas y deficiencias en los formatos en los que se codifican los datos. Estos aspectos pueden dividirse en: a) aquellos relativos a la falta de eficacia del formato, derivados de una mala elección de formato, y b) aquellos que ya están perfectamente caracterizados y 
descritos en la consistencia de formato tal y como se define en la norma de calidad ISO 19157 (ISO, 2013).

La calidad del formato elegido no es fácil de cuantificar; comprende, entre otros, aspectos como: a) que no se trate de un estándar abierto; b) que conlleve pérdidas de información; c) que sea poco eficaz debido normalmente a generar ficheros demasiado voluminosos; d) que no esté bien definido, ya sea por falta de documentación o por admitir variantes que exigen un acuerdo entre el emisor y el receptor de la información.

\section{Interoperabilidad de significado}

La correcta interpretación de unos datos transferidos depende esencialmente de la adecuada comprensión del modelo de realidad asociado, ya sea implícita o explícitamente. Por lo tanto, habría que considerar los riesgos derivados de: una deficiente calidad del modelo de aplicación, deficiencias del catálogo de objetos geográficos y de algo que suele olvidarse al gestionar la información geográfica vectorial, que es la deficiente selección de fenómenos del mundo real en función de la resolución:

- Deficiencias en el modelado. El Esquema de Aplicación es el componente más importante para transformar los datos en información, es decir, para interpretar los datos y dotarlos de significado. Puesto que el Esquema de Aplicación es el resultado de un proceso de selección de lo que resulta de interés a partir de lo real, de abstracción posterior y formalización en un lenguaje formalizado, como el Unified Modelling Language (UML), su calidad resulta difícil de describir cuantitativamente. Sin embargo, es posible identificar deficiencias de un esquema, como: clases sobreabundantes, redundancias, relaciones mal establecidas, complejidad excesiva, errores en las clases seleccionadas, las relaciones y los atributos, errores de sintaxis en UML, falta de detalle en el esquema, mala elección de los nombres y tipos de datos erróneos.

Los errores de modelado dificultan la interoperabilidad, generan problemas en la gestión y mantenimiento de los datos, dificultan la comprensión del problema y en general, son deficiencias profundas que actúan como fuentes de todo tipo de problemas (Muller, 1997).

Un punto interesante a tener en cuenta aquí es la más que probable posibilidad de superar estas deficiencias pasando a otra etapa de modelado de los datos basada en las Ontologías. Como técnica de modelado más avanzada y en la que se incorporan aspectos conceptuales de Inteligencia Artificial, debe necesariamente mejorar la fidelidad y coherencia del modelo producido. En ese caso, una línea de investigación interesante es la que consistente en la caracterización y descripción de la calidad de una Ontología (García et al., 2010), teniendo en cuenta las características particulares de las Ontologías geográficas. 
- Deficiencias en el catálogo de objetos. Da lugar a aspectos de la calidad que están un nivel de abstracción por encima de la exactitud temática y tienen que ver con la definición del catálogo de objetos geográficos. Incluye errores como: catálogo incompleto, solapes y huecos entre los objetos definidos, redundancias, errores en la estructura jerárquica de los tipos de objeto, errores formales y semántica (nombres y definiciones) poco clara y confusa, entre otros.

- Deficiencias en la selección por resolución. Una fuente de error que se suele olvidar, o al menos no tenerse suficientemente en cuenta. Cuando un técnico (surveyor) sale al campo a hacer un levantamiento a una resolución determinada sabe perfectamente qué fenómenos del mundo real debe recoger y cuáles debe obviar, en función de su tamaño real y su importancia. La misma situación se presenta al restituir fotogramétricamente, al digitalizar datos sobre una ortofoto y en general, en todos los procesos interactivos de adquisición de datos, como por ejemplo, la generalización. Incluye errores de selección por exceso, por defecto y falta de homogeneidad al aplicar los criterios de selección.

También habría que incluir aquí los aspectos geométricos de la resolución, como superficie mínima a representar, distancia mínima entre vértices, etc., susceptibles de ser descritos en la consistencia geométrica, y la resolución métrica de las coordenadas y el tiempo, incluidas en la consistencia de formato.

Es importante no olvidar estos aspectos, en realidad clásicos y de etiología bien conocida en los entornos digitales, en los que es fácil perder la noción de lo que significa trabajar en un modelo de una resolución determinada.

- Falta de Adaptabilidad Lingüística y Cultural. En este apartado es donde hay que incluir todos los aspectos relacionados con la Adaptabilidad Lingüística y Cultural (CLA por sus siglas en inglés, Cultural and Linguistic Adaptability), tal y como se define en ISO/IEC (2005): capacidad de un producto para tener en cuenta las necesidades usuales de cualquier grupo de usuarios y para ser internacionalizado manteniendo sus propiedades de portabilidad e interoperabilidad, es decir para ser adaptado a las características especiales de las culturas y lenguas naturales de una región geográfica dada, incluyendo sus reglas de uso. Es decir, multilingüismo ${ }^{5}$ y multiculturalismo. La CLA es un aspecto que puede evaluarse tanto para una IDE completa, como para cada uno de sus recursos: geoportales, clientes, servicios, conjuntos de datos, metadatos, etc.

5 No es un aspecto ocioso ni marginal en absoluto, dado que en realidad en contra de lo que puede parecer a primera vista, el multilingüismo es la situación más frecuente en un país y el monolingüismo, la excepción. En el mundo hay unas 7,000 lenguas y menos de 200 países; un caso extremo es el la República de Sudáfrica, con 11 idiomas oficiales. Por un lado, es lógico que las interfaces contemplen todos los idiomas oficiales del país más el inglés, pero al hablar de conjuntos de datos, hay que tener en cuenta que los nombres geográficos están en todos los idiomas, dialectos y variantes en uso en un país. 
La gestión del multilingüismo de interfaces de usuario, datos y metadatos es algo que puede solucionarse a nivel de aplicación, pero si pensamos en servicios web, tenemos que ser conscientes de que los servicios OGC no son multilingües.

A pesar de algunos intentos de considerar estos aspectos, como la introducción en las normas ISO 19100 que modelan datos y metadatos, el concepto de locale (un atributo que acompaña a las cadenas de texto e incluye el idioma en el que están escritas, la variante idiomática del país y el juego de caracteres empleado) parece que la CLA ha sido insuficientemente tenida en cuenta hasta ahora y es importante hacer un esfuerzo para incorporarla en los modelos de calidad.

Los aspectos indicados anteriormente afectan a los datos (modelo, catálogo, resolución, lenguaje), pero también hay aspectos semánticos en los servicios. Con estos aspectos nos referimos a entender plenamente qué es lo que ofrece un servicio. Por ello también se deben considerar:

- Interoperabilidad de significado de servicios. Aunque se puede englobar dentro de la conformidad de los servicios web con los estándares elegidos, se puede distinguir entre una conformidad meramente sintáctica, que garantiza que se cumplen todos los requisitos formales del estándar en cuanto a formatos, peticiones y parámetros, y una interoperabilidad que aseguraría además que se respeta el significado y sentido originales del estándar.

Por ejemplo, el significado y sentido originales del estándar WMS 3.1.0 (Open Geospatial Consortium) se pervierte, por ejemplo: a) cuando se utiliza el abstract (resumen) del documento de capabilities (capacidades) para codificar las restricciones de acceso, que deben ir en la etiqueta AccessConstraint; b) cuando se emplea la división en capas para organizar datos de distinta fecha, cuando para eso existe el parámetro TIME, o c) cuando se introduce una marca de aguas con un mensaje de copyright en el mapa que devuelve una petición GetMap, cuando de nuevo es la etiqueta AccessConstraint del capabilities el lugar estándar para codificar esa información.

- Falta de interoperabilidad de interfaces. Teniendo en cuenta que, más allá de la explotación automática de servicios web en remoto desde aplicaciones que los integran así en su propia lógica de aplicación, buena parte de la utilización de servicios web es llevada a cabo por usuarios humanos a través de interfaz de usuario, su calidad es un factor importante a tener en cuenta. 


\section{Interoperabilidad técnica}

Desde una aproximación basada en las IDE y la Arquitectura Orientada a Servicios, la interoperabilidad técnica depende de una serie de factores relacionados con las comunicaciones, como disponer del ancho de banda suficiente, el desempeño de las transferencias de datos, la disponibilidad y estabilidad de la conexión y la calidad de los servicios.

Efectivamente toda la funcionalidad de un sistema se puede conceptualizar como un servicio, incluyendo los servicios de descarga de datos cuando no se trabaja con servicios web sino fuera de línea y en local. Por lo tanto, buena parte de los aspectos de la calidad de una IDE relacionados con la interoperabilidad técnica se pueden repercutir en la calidad de servicio, descrita en el marco de la Directiva Europea INSPIRE (Comisión Europea, 2009b) por medio de tres parámetros establecidos de la siguiente manera:

- Disponibilidad o probabilidad de que el servicio no esté caído y responda, expresado habitualmente como un tanto por ciento de disponibilidad mensual o anual. Para determinarla se realizan peticiones cada seis minutos, que deben estar perfectamente definidas y descritas. Se exige al menos un $99 \%$ de disponibilidad anual excepto tiempos de parada técnica anunciados con una semana de antelación.

- Rendimiento o tiempo de respuesta, descrito como el tiempo que se tarda en recibir el primer bit de respuesta a una petición dada. Se emplean las mismas peticiones que sirven para determinar la disponibilidad para calcular medias anuales. El marco INSPIRE (Comisión Europea, 2009b) establece un límite de 5 segundos para un servicio WMS de visualización y 3 segundos para un servicio CSW de catálogo.

- Capacidad o posibilidad de responder un pico de peticiones de prueba, como por ejemplo 20 peticiones por segundo durante un minuto, manteniendo el rendimiento deseado (Comisión Europea, 2009b).

En cuanto a las interfaces habría que tener en cuenta tanto su interoperabilidad como su calidad, y en ese sentido sí se pueden encontrar algunos estudios sobre calidad de geoportales de una IDE, como son los de Rodríguez et al., 2015, 2017 y 2018. En ellos se definen una serie de aspectos de la calidad de un geoportal que atienden fundamentalmente a su apertura, es decir a la ausencia de barreras de todo tipo que limiten su uso y a la interoperabilidad, estrechamente ligada a la estandarización del recurso. Como consecuencia de los tres estudios mencionados, dedicados respectivamente a los geoportales españoles, los iberoamericanos y los europeos, lo que supone haber analizado 79 geoportales IDE nacionales o regionales, de España, Iberoamérica y Europa, y de haber realizado una encuesta para afinar la lista de 
indicadores entre 33 expertos IDE de todo el mundo, se llegó a seleccionar 19 parámetros para evaluar la calidad de geoportales, que no se incluyen aquí por estar ampliamente descritos en Rodríguez et al. (2018).

Hasta ahora se ha prestado cierta atención a la calidad de servicios web, pero muy poca a la calidad de geoportales, ya que apenas hay estudios al respecto más allá de los citados. En ese sentido, la lista de indicadores propuestos en esa referencia puede ser un buen punto de partida para analizar geoportales y hacer propuestas de mejora.

Finalmente y a otro nivel de abstracción, podría considerarse como otro aspecto de la calidad el grado de implementación de la Arquitectura Orientada a Servicios. Así, podría definirse algún indicador que describiese cuantitativamente hasta qué punto una organización productora de datos geográficos ha implementado realmente esta arquitectura examinando sus procesos de producción y viendo por ejemplo qué porcentaje de ellos desemboca en la publicación de servicios web.

\section{Conclusiones}

El ciclo de vida de las Infraestructuras de Datos Espaciales parece que presenta anomalías respecto de la evolución en el pasado de otras tecnologías similares, como los SIG, y es posible que se esté abriendo una brecha entre sus planteamientos y las vanguardias en el campo de la información geográfica. En consecuencia, nos parecen muy recomendables las líneas de investigación que traten de integrar esas nuevas tecnologías (datos enlazados, Big Data, Smart Cities, Internet of Things, Realidad Aumentada/Virtual, 3D, BIM, machine learning, etc.) y las tecnologías IDE, haciendo evolucionar tanto a las primeras como a las segundas. Están en juego los dos grandes pilares de las IDE: la interoperabilidad y la filosofía de datos y servicios abiertos.

Por otro lado, la implantación y evolución de los modelos de calidad IDE están llamadas a ser una de las palancas que potencien su desarrollo y progreso. En este trabajo se ha presentado un análisis de las aproximaciones utilizadas hasta ahora para la caracterización de la calidad global de una IDE, aspecto sobre el que se dispone de literatura (Randolf Pérez et al., 2015, Vancauwenberghe et al., 2018) sobre experiencias bajo diferentes enfoques, nacidos más de modelos teóricos que de experiencias prácticas.

Resulta difícil organizativamente y costoso realizar experiencias de aplicación de algunos de tales modelos, o de una variedad interesante de indicadores, en varias IDE y durante varios años, máxime cuando las IDE son sistemas complejos, sistemas de sistemas según (Béjar et al., 2009), que pueden tardar entre 10 y 15 años en alcanzar la madurez si se trata de un país o región compleja. Hay que tener en cuen- 
ta que el periodo de implementación previsto en la Directiva INSPIRE en Europa es de trece años (2007-2020).

Del análisis de la literatura sobre indicadores, se desprende que se pueden clasificar en cinco categorías: indicadores de acciones realizadas, de calidad de los componentes implementados, de uso, de impacto y de coste-beneficio.

En líneas generales, son más abundantes los análisis de calidad de una IDE basados en indicadores de acciones realizadas y de uso, pero su relación causa-efecto con la calidad final de una IDE es demasiado lejana. Por otro lado, resultan más interesantes por ser efecto casi directo de la calidad de una IDE los indicadores de impacto y de coste-beneficio, pero presentan el inconveniente de ser difícilmente objetivables debido a que los beneficios de una IDE son en su mayoría intangibles y se dan a muy largo plazo. En cambio, los indicadores de calidad de los componentes implementados resultan muy interesantes, si se considera un abanico suficientemente amplio de componentes de una IDE, ya que su calidad es causa directa de la calidad global de una IDE. Además, son relativamente fáciles de cuantificar objetivamente.

Para diseñar un modelo de calidad basado en indicadores de calidad de los componentes implementados proponemos tomar como base su característica más importante, la interoperabilidad, y para caracterizarla, un esquema de interoperabilidad avanzado y lo más completo posible, como el European Interoperability Framework v2 (Comisión Europea, 2017) y ampliarlo considerando el máximo abanico posible de aspectos de la interoperabilidad relevantes. A cada aspecto de la interoperabilidad contemplado en ese esquema ampliado, le correspondería un aspecto de la calidad.

Sin embargo, en muchos de los aspectos a tener en cuenta, se encuentran facetas poco exploradas y cuantificadas. De esas carencias nace la necesidad de abordar líneas de investigación que profundicen en los aspectos detallados en las secciones anteriores, especialmente investigación en la caracterización y determinación de la interoperabilidad legal, la interoperabilidad organizativa y la interoperabilidad de significado, toda vez que el resto de niveles y aspectos son relativamente bien conocidos.

Esa aproximación complementaría a las líneas de trabajo existentes en este campo, que siguen siendo oportunas y deseables, como los estudios de impacto y los análisis de coste-beneficio.

El Instituto Panamericano de Geografía e Historia puede ser una instancia privilegiada para fomentar este tipo de actividades y el foro idóneo tanto para compartir y discutir los resultados de las distintas líneas de investigación e innovación mencionadas, como para abordar la implementación de modelos de calidad para las IDE de la región. 


\section{Bibliografía}

AENOR (2003). "UNE 66175 Sistemas de gestión de la calidad - guía para la implantación de sistemas de indicadores", Madrid.

Ariza, F.J, y Rodríguez, A.F. (eds.) (2008). "Introducción a la Normalización en Información Geográfica: la familia ISO 19100. (130 pp.), Grupo de Investigación en Información Cartográfica, Universidad de Jaén, disponible en $<$ http://coello.ujaen.es/Asignaturas/pcartografica/Recursos/IntroduccionNormali zacion_IG_FamiliaISO_19100_v2.pdf>, consultado el 13 de agosto de 2018 .

Balciunas, A. and Rozanskas, E. (2016). National Benefits from INSPIRE Implementation. The Real Life Use Cases, en 2016 INSPIRE Conference, disponible en <http://inspire.ec.europa.eu/pr_searchx.cfm? $\mathrm{i}=5 \& \mathrm{id} \_$search $=50026>$, consultado en 13 de agosto de 2018.

Béjar, R.; Latre, M.A.; Nogueras-Iso, J.; Muro-Medrano, P.R. and Zarazaga-Soria, F.J. (2009). "Systems of Systems as a Conceptual Framework for Spatial Data Infrastructures", en International Journal of Spatial Data Infrastructure Research, vol. 4, JRC, ISPRA, Italia, 201-2017.

Comisión Europea (2009) "Decisión por la que se ejecuta la Directiva 2007/2/CE del Parlamento Europeo y del Consejo en lo que se refiere al seguimiento y los informes", disponible en $\quad<$ https://eurlex.europa.eu/LexUriServ/LexUriServ.do?uri=OJ:L:2009:148:0018:0026:ES:P DF>, consultado el 13 de agosto de 2018.

Comisión Europea (2009b). "Reglamento (CE) 976/2009 de la Comisión, de 19 de octubre de 2009 por el que se ejecuta la Directiva 2007/2/CE en lo que se refiere a los servicios de red", disponible en <http://eur-lex.europa.eu/legalcontent/ES/TXT/PDF/?uri=CELEX:32009R0976\&from=ES $>$, consultado el 13 de agosto de 2018.

Comisión Europea (2017). "European Interoperability Framework v2", disponible en <http://eur-lex.europa.eu/resource.html?uri=cellar:2c2f2554-0faf-11e7-8a3501aa75ed71a1.0017.02/DOC_3\&format=PDF $>$, consultado el 13 de agosto de 2018.

Comisión Europea (2017b) "Summary Report on Status of implementation or the INSPIRE Directive in EU', disponible en $<$ http://publications.jrc.ec.europa.eu/repository/bitstream/JRC109035/jrc109035 jrc109035_jrc_inspire_eu_summaryreport_online.pdf $>$, consultado el 13 de agosto de 2018 .

Crompvoets, J.; Rajabifard, A.; van Loenen, B. and Delgado, T. (eds.) (2008). “ $A$ Multi-View Framework to Assess SDIs", Space for Geo-Information, RGI, Wageningen University, Melbourne, Australia.

De Jong, C. (2016). "Cost-benefit analysis INSPIRE in the Netherlands", en INSPIRE Conference, disponible en 
$<$ http://inspire.ec.europa.eu/pr_searchx.cfm?i=5\&id_search $=50025>$, consultado el 13 de agosto de 2018.

EEA-JRC (2014). "Mid-term evaluation report on INSPIRE implementation", disponible en <https://www.eea.europa.eu/publications/midterm-evaluation-reporton-inspire-implementation>, consultado el 13 de agosto de 2018.

García, J.; García-Peñalvo, F.J. and Therón, R. (2010). "A Survey on Ontology Metrics", en Knowledge Management, Information Systems, E-learning and Sustainability Research, Springler, 22-27.

Giff, G. and Crompvoets, J. (2008). "Performance Indicators: a tool to Support Spatial Data Infraestructure assessment", Computers, Environment and Urban Systems, vol. 32, Elsevier, Amsterdam, Países Bajos, 365-376.

GSDI (2012). SDI Cookbook, disponible en $<$ http://gsdiassociation.org/images/publications/cookbooks/SDI_Cookbook_fro m_Wiki_2012_update.pdf $>$, consultado el 13 de agosto de 2018.

ISO/IEC (2005). "TR 9764:2005 Information technology - Guidelines, methodology and reference criteria for cultural and linguistic adaptability in information technology products", ISO Ginebra.

Kelm K.; Probert, M. and Tonchovska (2017). "Creating a Spatial Data Infrastructure Diagnostic Tool", World Bank Conference on Land and Poverty, marzo 2024, 2017, Washington DC.

Maganto, A.S.; Rodríguez, A.F. y Bernabé, M.A. (2012). "Componentes de una IDE”, en Bernabé-Poveda, M.A. y López-Vázquez, C.M., Fundamentos de las Infraestructuras de Datos Espaciales, UPM Press Serie Científica, Madrid. España, pp. 55-65.

Mani, A. and Nagarajan, A. (2002). "Understanding quality of service for Web Services. Improving the performance of tour Web services", IBM, disponible en $<$ https://www.ibm.com/developerworks/library/ws-quality/index.html>, consultado el 13 de gusto de 2018.

Martín, A.; Contreras, J.J.; Salinas, E.; Valverde, P.; Balbi, R.; Medeiros, V.E.; Vianey, A.; Fajardo, A.; Guerrón, P.; Tene, E.; Sámuels, E.; Agrazal, A.; Flores, R.; Avilés, W.; Parcher, J.; Lugo, R.; Blanco, L.M. y Rodríguez, A.F. (2017). "El Mapa Integrado Andino del Norte", en Revista Cartográfica, núm. 94, enero-junio, Instituto Panamericano de Geografía e Historia, 2017, 11-27.

Morera, C.; Carrasquilla, O.; Rey, D. y Guimet, J. (2012). "Evaluación de una IDE desde su caracterización hasta su impacto en la sociedad", en Bernabé-Poveda, M.A. y López-Vázquez, C.M., Fundamentos de las Infraestructuras de Datos Espaciales, UPM Press Serie Científica, Madrid, España, 443-452.

Mueller, P.A. (1997). "Modelado con objetos UML”, editorial Eyrolles, París. 
Nativi, S.; Craglia, M. and Pealman, J. (2012). "The Brokering Approach for Multidisciplinary Interoperability: A Position paper", in International Journal of Spatial Infraestructures Research, vol. 4, 1-15.

Norori, M.; Williams, C.; Torres, E.; Salazar, L.; Sáncho, E.; Flores, E.; Gómez, B. I.; Benítez, F.; Menendez, R.; Núñez, M.; Martínez, A.; Parcher, J.; Roberto, L.; Zavala, E.; Ramírez, N.; Pérez, J.; Morales, C.; Sámuels, E. y Mondragón, R. (2013). "Proceso participativo de producir un Mapa Integrado de Centroamérica y Sur de México”, en Revista Cartográfica, núm. 89, enero-diciembre, Instituto Panamericano de Geografía e Historia, 47-60.

Open Geospatial Consortium (2006). “OpenGIS Web Map Server Implementation specification $\quad \mathrm{V} \quad 1.3 .0 ”, \quad$ disponible $<$ http://www.opengeospatial.org/standards/wms $>$, consultado el 20 de marzo de 2018.

Orden Ejecutiva 12906. "Coordinating Geographic Data Acquisition and Access: The National Spatial Data Infrastructure” (1994), Presidente de Estado Unidos, disponible en <https://www.archives.gov/files/federal-register/executiveorders/pdf/12906.pdf $>$, consultado el 13 de agosto de 2018.

Randolf Pérez, D.; Ballari, D. y Vilches-Blázquez, L. (2015). "Participación y dinamicidad en las Infraestructuras de Datos Espaciales: una propuesta de indicadores para medir su impacto en la sociedad”, en Revista Cartográfica, núm. 90, enero-diciembre, Instituto Panamericano de Geografía e Historia, 175-191.

Rodríguez, A.F.; Abad, P.; Sánchez, A.; Juanatey, M. y Cevidanes, A. (2015). "Interoperabilidad práctica de los geoportales de la IDEE” presentación realizada en las JIIDE 2015, disponible en $<$ http://www.idee.es/resources/presentaciones/JIIDE15/20151104/18_Interopera bilidadPracticadeGeoportales.pdf $>$, consultado el 13 de agosto de 2018.

Rodríguez, A.F. (1993). "Los SIG en España: un campo sin cartografiar”, Topografia y Cartografía, núm. 55, 25-41.

Rodríguez, A.F.; Abad, P.; Sánchez, A.; Juanatey, M. y Cevidanes, A. (2018). "Aproximación a una metodología de evaluación de la calidad de geoportales IDE”, Revista de Cartografía, núm. 95, número monográfico sobre geoportales, julio-diciembre, Instituto Panamericano de Geografía e Historia, 17-42.

Rodríguez A.F.; Sevilla C.; Abad P.; López, E. y Sánchez A. (2013). "Panorama de la estandarización en el campo de la información geográfica: la importancia de los estándares abiertos”, CIGMA, México.

Rodríguez, A.F.; Abad, P.; Sánchez, A.; Juanatey, M. y Cevidanes, A. (2017). "Towards a Methodology to evaluate the Quality of SDI Geoportals" presentación realizada en el Congreso INSPIRE 2017, disponible en $<$ https://inspire.ec.europa.eu/sites/default/files/presentations/2017InspireConfere nceGeoportalsAFRodriguez.pdf $>$, consultado el 13 de agosto de 2018 . 
Rodríguez, A.F., López, E., Cabria, A., Rodríguez, J.M. y Vivas, P. (2017b). "El nuevo Esquema Europeo de Interoperabilidad y las IDE", en MAPPING, vol. 26, noviembre-diciembre, 186, 6-11.

UN-GGIM (2018). "Integrated Geospatial Information Framework. A strategic guide to develop and strengthen National Geospatial Information Management. Part 1: Overarching Strategic Framework", disponible en $<$ http://ggim.un.org/meetings/GGIM-committee/8thSession/documents/Part\%201-IGIF-Overarching-Strategic-Framework24July2018.pdf $>$, consultado el 13 de agosto de 2018.

Vandenbroucke, D. (2012). "Did we approach the objectives of INSPIRE?", INSPIRE Conference, disponible en $<$ http://inspire.jrc.ec.europa.eu/events/conferences/inspire_2012/presentations/1 83.pdf $>$, consultado el 13 de agosto de 2018.

Vancauwenberghe, G.; Valeckaite, K.; van Loenen, B. and Welle Donker, Frederika (2018). "Assesing the Openness of Spatial Data Infrastructures (SDI): Towards a Map of Open SDI", International Journal of Spatial Data Infrastructures Research, 13, 88-100.

Welle Donker, F. and B. van Loenen (2016). "How to assess the success of the open data ecosystem?" International Journal of Digital Earth, 10 (3), 284-306.

World Wide Web Consortium (2003). "QoS for Web Services: Requirements and Possible Approaches". W3C Working Group Note, disponible en $<$ http://www.w3c.or.kr/kr-office/TR/2003/ws-qos/>, consultado el 13 de agosto de 2018.

Worl Wide Web Consortium (2017). "Spatial Data on the Web Best Practices". W3C Working Group Note, disponible en $<$ https://www.w3.org/TR/sdw-bp/>, consultado el 13 de agosto de 2018. 